\title{
Effect of Combination Therapy of Canagliflozin Added to Teneligliptin Monotherapy in Japanese Subjects with Type 2 Diabetes Mellitus: A Retrospective Study
}

\author{
Yoshiro Fushimi, Atsushi Obata $(\mathbb{D}$, Junpei Sanada, Yuichiro Iwamoto, Akiko Mashiko, \\ Megumi Horiya, Akiko Mizoguchi-Tomita, Momoyo Nishioka, Yuki Kan, Tomoe Kinoshita, \\ Seizo Okauchi, Hidenori Hirukawa $\mathbb{D}$, Kenji Kohara, Fuminori Tatsumi, \\ Masashi Shimoda (D), Shuhei Nakanishi, Tomoatsu Mune, Kohei Kaku, \\ and Hideaki Kaneto
}

Department of Diabetes, Endocrinology and Metabolism, Kawasaki Medical School, Japan

Correspondence should be addressed to Atsushi Obata; obata-tky@med.kawasaki-m.ac.jp

Received 28 October 2019; Revised 10 February 2020; Accepted 20 March 2020; Published 2 April 2020

Academic Editor: Ulrike Rothe

Copyright (C) 2020 Yoshiro Fushimi et al. This is an open access article distributed under the Creative Commons Attribution License, which permits unrestricted use, distribution, and reproduction in any medium, provided the original work is properly cited.

\begin{abstract}
Recently, dipeptidyl peptidase-4 (DPP-4) inhibitors and sodium-glucose cotransporter 2 (SGLT2) inhibitors have been very often used in subjects with type 2 diabetes mellitus (T2DM). In addition, combination drugs of both inhibitors have attracted much attention in aspects of its cost-effectiveness and improvement of patients' adherence. However, it is still poorly understood which factors are related to the efficacy of SGLT2 inhibitors as add-on therapy to DPP-4 inhibitors. Therefore, we aimed to elucidate in which type of individuals and/or under which conditions canagliflozin as add-on therapy to teneligliptin could exert more beneficial effects on glycemic control and/or renal protection. We retrospectively analyzed 56 Japanese subjects with $\mathrm{T} 2 \mathrm{DM}$ in the real-world clinical practice. Three months after starting the combination therapy, the change of HbA1c $(\Delta \mathrm{HbA} 1 \mathrm{c})$ was strongly related to $\mathrm{HbA1c}$ levels at baseline. As expected, serum glucagon level was increased after starting the combination therapy. Interestingly, however, the change of glucagon levels ( $\Delta$ glucagon) was not related to HbAlc levels at baseline, $\Delta \mathrm{HbA1c}$, and other parameters, which indicated that the increase of glucagon did not clinically affect the effectiveness of combination therapy. In addition, the change of urinary albumin excretion ( $\triangle \mathrm{UAE}$ ) was negatively correlated with systolic blood pressure and $\mathrm{HbA1c}$ levels at baseline and positively correlated with the change of systolic blood pressure $(\Delta \mathrm{sBP})$ in univariate analysis. Furthermore, in multivariate analysis, only $\triangle \mathrm{sBP}$ was the independent factor associated with $\triangle \mathrm{UAE}$. Taken together, canagliflozin as add-on therapy to teneligliptin improves glycemic control in a $\Delta$ glucagon-independent manner and reduces $\mathrm{UAE}$ in a $\triangle \mathrm{sBP}$-dependent manner in Japanese subjects with T2DM.
\end{abstract}

\section{Introduction}

Recently, DPP-4 inhibitors are commonly used in various individuals with type 2 diabetes mellitus (T2DM) from the young to the elderly because of their good glucose-lowering effect and safety including low risk of hypoglycaemia. DPP4 inhibitors promote insulin secretion and suppress glucagon secretion by inhibiting the degradation of glucagon-like peptide-1 [1]. SGLT2 inhibitors, which emerged in clinical practice after DPP-4 inhibitors, improve glycemic control by reducing filtered renal glucose reabsorption from the proximal tubule and promoting excretion of excess glucose, which is a totally different mechanism compared with DPP4 inhibitors [2]. SGLT2 inhibitors are promising drugs in view of reduction of glucose toxicity, body weight, and insulin resistance $[3,4]$. In addition, large clinical trials have 
shown that SGLT2 inhibitors improved 3-point major adverse cardiovascular events (MACE) and renal outcomes [5-7], which were not observed with DPP-4 inhibitors. Since DPP-4 inhibitors and SGLT2 inhibitors have such different mechanisms of action and the risk of hypoglycaemia with both medications is pretty low, it seems that the combination therapy of both inhibitors is very promising. In fact, large clinical trials have shown the efficacy and safety of combination therapy in Japanese individuals with type 2 diabetes $[8,9]$. In addition, very recently, combination drugs of both inhibitors became available in clinical practice and have drawn much attention in aspects of its cost-effectiveness and enhancement of patients' adherence. However, it is still poorly understood which factors and/or features of individuals mainly contribute to the glucose-lowering and/or renal protection effects of such combination therapy in realworld clinical practice. Therefore, we aimed to address the answer of these questions in this study.

\section{Methods}

2.1. Study Design. The present study was retrospectively carried out with outpatients in the Division of Diabetes, Endocrinology and Metabolism in Kawasaki Medical School from February to December in 2018. The subjects who had been taking teneligliptin $(20 \mathrm{mg} /$ day $)$ for longer than 6 months without any other medication changes were included.

The participants were those who fulfilled the following criteria: (i) without severe renal dysfunction (eGFR $<30 \mathrm{~mL} / \mathrm{min} / 1.73 \mathrm{~m}^{2}$ ); (ii) without any insulin and/or GLP-1RA usage; (iii) without infectious disease, malignancy, or diabetic coma; and (iv) not using steroid and/or immunosuppressant medications. A total of 91 participants ( 48 males, 43 females) were enrolled in the present study, and canagliflozin (100 mg/day) was added on to the subjects by each doctor's judgement. As a result, 56 out of 91 participants received canagliflozin $(100 \mathrm{mg} /$ day $)$ and the remaining 35 participants continued teneligliptin. After starting canagliflozin add-on therapy for 3 months, blood and urinary samples after overnight fasting were obtained from all patients for measuring the multiple metabolic parameters. Serum glucagon levels were measured by SRL (Glucagon ELISA, Cosmic, Mercodia AB, Sylveniusgatan 8A, 75450 Uppsala, Sweden). The study protocol was approved by the institutional review board of Kawasaki Medical School (No. 3236), and the study was carried out in accordance with the Declaration of Helsinki, and we provided public information on the study via the Internet, instead of obtaining informed consent from each patient. We carried out data collection for variables such as type of medication as well as biochemical data.

2.2. Evaluation of Body Composition Parameters. Body composition variables were measured by a bioelectrical impedance body composition analyzer InBody 770 (InBody Japan Inc., Japan). Measurements were performed with the participants barefoot and in minimal clothing without any metallic materials. Body weight, total body water, intracellular and extracellular water, body fat mass, percent body of fat, soft lean mass, and skeletal muscle mass were measured before and after canagliflozin add-on therapy.

2.3. Statistical Analysis. All data were analyzed using JMP version 11.0 and are presented as mean \pm standard deviation (SD) or number (percentage). Changes in parameters from the baseline values in the same patient were evaluated using Wilcoxon singed-rank test. Baseline parameters and changes of various parameters of the teneligliptin continued group and canagliflozin add-on group were analyzed using Wilcoxon rank sum test. To examine which factors are associated with change $(\Delta)$ in HbAlc, glucagon, and UAE, we carried out Spearman's rank correlation coefficient test. Furthermore, to examine which factors independently determine $\triangle \mathrm{UAE}$ after 12 weeks of canagliflozin add-on therapy, we carried out multiple regression analysis. $p$ value $<0.05$ was considered to be statistically significant.

\section{Results}

3.1. Clinical Characteristics of the Study Subjects. As shown in Table 1, clinical characteristics of the study subjects were as follows: in the teneligliptin continued group: age, $68.3 \pm$ 11.4 years old; number (male/female), 35 (21/14); duration of diabetes, $17.8 \pm 12.9$ years; body weight, $63.1 \pm 16.4 \mathrm{~kg}$; BMI, $24.3 \pm 5.4 \mathrm{~kg} / \mathrm{m}^{2}$; HbA1c, $6.9 \pm 0.6 \%$; systolic blood pressure, $125 \pm 13 \mathrm{mmHg}$; diastolic blood pressure, $71 \pm$ $10 \mathrm{mmHg}$; triglyceride, $114 \pm 51 \mathrm{mg} / \mathrm{dL} ; \mathrm{LDL}$ cholesterol, $96.6 \pm 22.8 \mathrm{mg} / \mathrm{dL} ; \quad \mathrm{HDL}$ cholesterol, $54.1 \pm 14.1 \mathrm{mg} / \mathrm{dL}$; and uric acid, $5.6 \pm 1.3 \mathrm{mg} / \mathrm{dL}$. The prevalence rates of subjects with diabetic microangiopathy were as follows: peripheral neuropathy $40 \%$, retinopathy $26 \%$, and nephropathy stage $160 \%$, stage $220 \%$, and stage $320 \%$, respectively. The prevalence of subjects with history of myocardial infarction and/or stroke was 34\%. The prevalence of concomitant medication was as follows: DPP-4 inhibitor 100\% (teneligliptin $20 \mathrm{mg} /$ day), biguanide $46 \%$, sulfonylurea or glinide $37 \%$, thiazolidine $34 \%$, and $\alpha$-glucosidase inhibitor $29 \%$. Insulin and/or GLP-1 receptor agonist users were not included in this study.

On the other hand, in the canagliflozin add-on group, the clinical characteristics of the study subjects were as follows: age, $61.6 \pm 13.5$ years old; number (male/female), 56 (27/29); duration of diabetes, $11.3 \pm 8.2$ years; body weight, $72.3 \pm 20.2 \mathrm{~kg} ; \mathrm{BMI}, 28.6 \pm 6.7 \mathrm{~kg} / \mathrm{m}^{2}$; HbA1c, $7.6 \pm 1.5 \%$; systolic blood pressure, $133 \pm 20 \mathrm{mmHg}$; diastolic blood pressure, $80 \pm 14 \mathrm{mmHg}$; triglyceride, $157 \pm 143 \mathrm{mg} / \mathrm{dL}$; LDL cholesterol, $103.4 \pm 32.9 \mathrm{mg} / \mathrm{dL}$; HDL cholesterol, $52.2 \pm 15.0 \mathrm{mg} / \mathrm{dL}$; and uric acid, $5.4 \pm 1.5 \mathrm{mg} / \mathrm{dL}$. The prevalence rates of subjects with diabetic microangiopathy were as follows: peripheral neuropathy $14 \%$, retinopathy $12 \%$, and nephropathy stage $173 \%$, stage $218 \%$, and stage $39 \%$, respectively. The prevalence of subjects with history of myocardial infarction and/or stroke was $9 \%$. The prevalence of concomitant medication was as follows: DPP-4 inhibitor 100\% (teneligliptin $20 \mathrm{mg} /$ day), biguanide $46 \%$, sulfonylurea or glinide $19 \%$, thiazolidine $20 \%$, and $\alpha$ glucosidase inhibitor $2 \%$. As you can see in Table 1 , the teneligliptin continued group presented significantly older 
TABLE 1: Efficacy of canagliflozin add-on therapy on various metabolic parameters.

\begin{tabular}{|c|c|c|c|c|c|c|}
\hline \multirow{3}{*}{$\begin{array}{l}\text { Parameters } \\
\text { Group } \\
\text { Age (years) }\end{array}$} & Baseline & 12 weeks & \multirow[t]{2}{*}{$p$} & Baseline & 12 weeks & \multirow[t]{2}{*}{$p$} \\
\hline & \multicolumn{2}{|c|}{ Teneligliptin continued group } & & \multicolumn{2}{|c|}{ Canagliflozin add-on group } & \\
\hline & $68.3 \pm 11.4$ & - & - & $61.6 \pm 13.5^{*}$ & - & - \\
\hline Gender $(\mathrm{M} / \mathrm{F})$ & $21 / 14$ & - & - & $27 / 29^{*}$ & - & - \\
\hline DM duration (years) & $17.8 \pm 12.9$ & - & - & $11.3 \pm 8.2^{*}$ & - & - \\
\hline Body weight (kg) & $63.1 \pm 16.4$ & $63.8 \pm 16.6$ & n.s. & $72.3 \pm 20.2^{*}$ & $71.0 \pm 20.2$ & $<0.0001$ \\
\hline BMI $\left(\mathrm{kg} / \mathrm{m}^{2}\right)$ & $24.3 \pm 5.4$ & $24.5 \pm 5.4$ & n.s. & $28.6 \pm 6.7^{*}$ & $27.7 \pm 6.7$ & $<0.0001$ \\
\hline Fat mass $(\mathrm{kg})$ & - & - & - & $27.7 \pm 14.2$ & $26.3 \pm 14.2$ & $<0.0001$ \\
\hline Visceral fat area $\left(\mathrm{cm}^{2}\right)$ & - & - & - & $137 \pm 62$ & $129 \pm 64$ & $<0.0001$ \\
\hline Skeletal muscle mass $(\mathrm{kg})$ & - & - & - & $25.1 \pm 7.5$ & $24.9 \pm 7.5$ & 0.047 \\
\hline FPG $(\mathrm{mg} / \mathrm{dL})$ & $138 \pm 22$ & $134 \pm 27$ & n.s. & $166 \pm 51^{*}$ & $139 \pm 27$ & $<0.0001$ \\
\hline HbAlc (\%) & $6.9 \pm 0.6$ & $6.9 \pm 0.6$ & n.s. & $7.6 \pm 1.5^{*}$ & $6.9 \pm 0.7$ & $<0.0001$ \\
\hline $\mathrm{IRI}(\mu \mathrm{IU} / \mathrm{mL})$ & - & - & - & $12.3 \pm 9.7$ & $16.4 \pm 20.2$ & n.s. \\
\hline Glucagon $(\mathrm{pg} / \mathrm{mL})$ & - & - & - & $149 \pm 40$ & $163 \pm 53$ & 0.0010 \\
\hline IRI/glucagon & - & - & - & $0.09 \pm 0.07$ & $0.09 \pm 0.09$ & n.s. \\
\hline Systolic BP (mmHg) & $125 \pm 13$ & $124 \pm 13$ & n.s. & $133 \pm 20^{*}$ & $129 \pm 17$ & 0.0041 \\
\hline Diastolic BP (mmHg) & $71 \pm 10$ & $70 \pm 10$ & n.s. & $80 \pm 14^{*}$ & $78 \pm 12$ & 0.032 \\
\hline AST (U/L) & $24.2 \pm 9.5$ & $22.4 \pm 8.1$ & n.s. & $28.4 \pm 18.0$ & $26.3 \pm 11.2$ & n.s. \\
\hline $\operatorname{ALT}(\mathrm{U} / \mathrm{L})$ & $20.7 \pm 12.1$ & $19.4 \pm 12.4$ & n.s. & $27.9 \pm 12.7^{*}$ & $26.4 \pm 9.0$ & 0.031 \\
\hline$\gamma \mathrm{GTP}(\mathrm{U} / \mathrm{L})$ & $30.3 \pm 35.2$ & $29.4 \pm 30.1$ & n.s. & $46.8 \pm 51.6^{*}$ & $39.3 \pm 23.9$ & 0.0005 \\
\hline Cre $(\mathrm{mg} / \mathrm{dL})$ & $0.8 \pm 0.3$ & $0.8 \pm 0.3$ & n.s. & $0.7 \pm 0.2$ & $0.7 \pm 0.2$ & n.s. \\
\hline BUN (mg/dL) & $17.9 \pm 5.3$ & $17.2 \pm 5.7$ & n.s. & $15.2 \pm 4.5^{*}$ & $17.5 \pm 4.5$ & $<0.0001$ \\
\hline eGFR $\left(\mathrm{mL} / \mathrm{min} / 1.73 \mathrm{~m}^{2}\right)$ & $69.7 \pm 18.7$ & $72.9 \pm 21.0$ & n.s. & $81.2 \pm 21.7^{*}$ & $79.7 \pm 23.9$ & n.s. \\
\hline Triglyceride (mg/dL) & $114 \pm 51$ & $106 \pm 44$ & n.s. & $157 \pm 143$ & $163 \pm 186$ & n.s. \\
\hline HDL cholesterol (mg/dL) & $54.1 \pm 14.1$ & $54.1 \pm 15.0$ & n.s. & $52.2 \pm 15.0$ & $50.2 \pm 12.0$ & n.s. \\
\hline LDL cholesterol (mg/dL) & $96.6 \pm 22.8$ & $92.4 \pm 21.8$ & n.s. & $103.4 \pm 32.9$ & $103.5 \pm 29.2$ & n.s. \\
\hline Ketone body $(\mu \mathrm{mol} / \mathrm{L})$ & - & - & - & $114 \pm 117$ & $141 \pm 154$ & 0.024 \\
\hline Uric acid (mg/dL) & $5.6 \pm 1.3$ & $5.4 \pm 1.4$ & n.s. & $5.4 \pm 1.5$ & $4.9 \pm 0.8$ & $<0.0001$ \\
\hline Urinary albumin excretion (mg/g. Cre) & $148 \pm 285$ & - & - & $101 \pm 228$ & $72 \pm 172$ & 0.020 \\
\hline Urinary glucose excretion (mg/g. Cre) & - & - & - & $71 \pm 478$ & $2672 \pm 2126$ & $<0.0001$ \\
\hline
\end{tabular}

${ }^{*}$ Teneligliptin continued group $v$ s. canagliflozin add-on group at baseline (Wilcoxon rank sum test), $p<0.05$.

age, longer duration of diabetes, and lower body weight, FPG, HbA1c, blood pressure, ALT, $\gamma$ GTP, and eGFR at baseline compared with the canagliflozin add-on group. No severe adverse events were observed in this study.

3.2. Effects of Combination Therapy of Canagliflozin Added to Teneligliptin Monotherapy on Various Metabolic Parameters. Three months after starting canagliflozin as add-on therapy to teneligliptin, body weight, BMI, HbA1c, fasting blood glucose levels, both systolic and diastolic blood pressures, urinary albumin excretion (UAE), urinary acid, and transaminase were all significantly reduced compared to those before such combination therapy (Table 1). Body weight was decreased by approximately $1.3 \mathrm{~kg}(72.3 \pm 20.2 \mathrm{~kg}$ to $71.0 \pm 20.2 \mathrm{~kg}$, $p<0.0001)$, and HbAlc levels were decreased by approxi- mately $0.7 \%(7.6 \pm 1.5 \%$ to $6.9 \pm 0.7 \%, p<0.0001)$. In body composition, fat mass was significantly decreased $(27.7 \pm 14.2 \mathrm{~kg}$ to $26.3 \pm 14.2 \mathrm{~kg}, p<0.0001$, respectively) accompanied with slight skeletal muscle mass reduction (decrease by approximately $0.2 \mathrm{~kg}$ ), which was quite little compared to the reduction of fat mass (decrease by approximately $1.4 \mathrm{~kg}$ ). Both systolic and diastolic blood pressures were decreased after starting canagliflozin as addon therapy to teneligliptin $(133 \pm 20 \mathrm{mmHg}$ to $129 \pm 17$ $\operatorname{mmHg}(p=0.0041)$ and $80 \pm 14 \mathrm{mmHg}$ to $78 \pm 12 \mathrm{mmHg}$ $(p=0.032)$, respectively). Urinary albumin excretion was also significantly reduced after such combination therapy $(101 \pm 228 \mathrm{mg} / \mathrm{g}$. Cre to $72 \pm 172 \mathrm{mg} / \mathrm{g}$. Cre, $p=0.020)$. In contrast, ketone body level was significantly increased $(114 \pm 117 \mu \mathrm{mol} / \mathrm{L} \quad$ to $\quad 141 \pm 154 \mu \mathrm{mol} / \mathrm{L}, \quad p=0.024)$. 
TABLE 2: Changes of various parameters between baseline and 3 months later.

\begin{tabular}{|c|c|c|c|}
\hline Parameters & Teneligliptin continued group & Canagliflozin add-on group & $p$ \\
\hline$\Delta$ Body weight $(\mathrm{kg})$ & $+0.7 \pm 1.5$ & $-1.3 \pm 1.9$ & $<0.0001$ \\
\hline$\Delta \mathrm{BMI}\left(\mathrm{kg} / \mathrm{m}^{2}\right)$ & $+0.2 \pm 0.6$ & $-0.9 \pm 0.7$ & $<0.0001$ \\
\hline$\Delta \mathrm{FPG}(\mathrm{mg} / \mathrm{dL})$ & $-4.3 \pm 22.1$ & $-27.3 \pm 40.3$ & 0.0003 \\
\hline$\Delta \mathrm{HbA} 1 \mathrm{c}(\%)$ & $-0.02 \pm 0.4$ & $-0.7 \pm 0.8$ & $<0.0001$ \\
\hline$\Delta$ Systolic BP (mmHg) & $-0.4 \pm 12.2$ & $-4.6 \pm 10.5$ & n.s. \\
\hline$\Delta$ Diastolic BP (mmHg) & $-0.2 \pm 9.7$ & $-2.6 \pm 9.4$ & n.s. \\
\hline$\Delta \mathrm{AST}(\mathrm{U} / \mathrm{L})$ & $-1.8 \pm 6.3$ & $-2.1 \pm 9.1$ & n.s. \\
\hline$\Delta \mathrm{ALT}(\mathrm{U} / \mathrm{L})$ & $-1.3 \pm 6.4$ & $-1.5 \pm 11.6$ & n.s. \\
\hline$\Delta \gamma \mathrm{GTP}(\mathrm{U} / \mathrm{L})$ & $-0.9 \pm 9.8$ & $-7.5 \pm 22.5$ & 0.03 \\
\hline$\Delta \operatorname{Cre}(\mathrm{mg} / \mathrm{dL})$ & $-0.02 \pm 0.07$ & $+0.02 \pm 0.07$ & 0.01 \\
\hline$\Delta \mathrm{BUN}(\mathrm{mg} / \mathrm{dL})$ & $-0.7 \pm 3.9$ & $+2.3 \pm 3.3$ & 0.001 \\
\hline$\Delta \mathrm{eGFR}\left(\mathrm{mL} / \mathrm{min} / 1.73 \mathrm{~m}^{2}\right)$ & $+3.2 \pm 8.6$ & $-1.5 \pm 8.8$ & 0.04 \\
\hline$\Delta$ Triglyceride $(\mathrm{mg} / \mathrm{dL})$ & $-8.0 \pm 40.7$ & $+6.2 \pm 70.2$ & n.s. \\
\hline$\Delta$ HDL cholesterol $(\mathrm{mg} / \mathrm{dL})$ & $-0.02 \pm 4.8$ & $-2.1 \pm 10.6$ & n.s. \\
\hline$\Delta \mathrm{LDL}$ cholesterol $(\mathrm{mg} / \mathrm{dL})$ & $-4.2 \pm 13.6$ & $+0.1 \pm 19.6$ & n.s. \\
\hline$\Delta \operatorname{Uric}$ acid $(\mathrm{mg} / \mathrm{dL})$ & $-0.2 \pm 0.6$ & $-0.6 \pm 0.7$ & 0.02 \\
\hline
\end{tabular}

Furthermore, interestingly, the serum glucagon level was significantly increased after starting the combination therapy $(149 \pm 40 \mathrm{pg} / \mathrm{mL}$ to $163 \pm 53 \mathrm{pg} / \mathrm{mL}, p=0.0010)$.

On the other hand, no variable changes were observed in the teneligliptin continued group between baseline and after three months. We next compared the changes of various parameters between baseline and 3 months later. As shown in Table 2, the decreased changes of body weight, BMI, FPG, and HbA1c were all significantly larger in the canagliflozin add-on group compared with the teneligliptin continued group. In addition, the decreased changes of $\gamma \mathrm{GTP}$, eGFR, and uric acid were also significantly larger accompanied with the significant increased change of Cre in the canagliflozin add-on group.

3.3. Search for Possible Factors Which Are Strongly Associated with Glucose-Lowering Effect and the Increase of Serum Glucagon Levels after Combination Therapy. We next investigated which factors were strongly associated with the glucose-lowering effect of canagliflozin as add-on therapy to teneligliptin. First, since HbA1c levels were significantly reduced after starting the combination therapy (Table 1), we evaluated which factors were closely associated with $\triangle \mathrm{HbA} 1 \mathrm{c}$ in univariate analysis. However, only HbA1c levels at baseline were strongly associated with $\triangle \mathrm{HbA} 1 \mathrm{c}$ (data not shown). Next, since serum glucagon levels were significantly elevated after the combination therapy (Table 1), we analyzed which factor levels at baseline were strongly associated with $\Delta$ glucagon. As shown in Table 3, however, $\Delta$ glucagon was not significantly related to HbAlc, IRI, and FPG at baseline in the univariate analysis. In addition, we examined the possible association of $\Delta$ glucagon with the change of various parameters such as $\triangle \mathrm{HbAlc}, \triangle \mathrm{IRI}$, and $\triangle \mathrm{FPG}$. However, there were no factors which were strongly associ-
TABLE 3: Various parameters associated with $\Delta$ glucagon in univariate analyses.

\begin{tabular}{lcc}
\hline Comparison with baseline value & $\rho$ & $p$ \\
Age (years) & -0.0088 & n.s. \\
Gender & 0.0426 & n.s. \\
Baseline FPG $(\mathrm{mg} / \mathrm{dL})$ & -0.0936 & n.s. \\
Baseline $\mathrm{HbAlc}(\%)$ & -0.1502 & n.s. \\
Baseline IRI $(\mu \mathrm{IU} / \mathrm{mL})$ & 0.1216 & n.s. \\
\hline Comparison with change of each value & $\rho$ & $p$ \\
Age $($ years $)$ & -0.0088 & n.s. \\
Gender & 0.0426 & n.s. \\
$\Delta$ FPG $(\mathrm{mg} / \mathrm{dL})$ & 0.0582 & n.s. \\
$\Delta \mathrm{HbA} 1 \mathrm{c}(\%)$ & 0.1366 & n.s. \\
$\Delta \mathrm{IRI}(\mu \mathrm{IU} / \mathrm{mL})$ & -0.1756 & n.s. \\
\hline
\end{tabular}

ated with $\Delta$ glucagon, indicating that the elevation of glucagon levels did not affect the glucose-lowering effect in such combination therapy.

3.4. Search for Possible Factors Which Are Strongly Associated with the Reduction of Urinary Albumin Excretion (UAE) after Combination Therapy. Since UAE was significantly reduced after starting canagliflozin as add-on therapy to teneligliptin (Table 1), we next investigated which factors were closely associated with the reduction of UAE ( $\triangle \mathrm{UAE}$ ). First, in the univariate analysis, $\triangle \mathrm{UAE}$ was significantly related to sBP, HbA1c levels at baseline, and $\triangle \mathrm{sBP}$ (Table 4). Next, to elucidate the independent factors determining the efficacy of combination therapy, we performed multivariate analysis. To avoid the possible influence of age or gender, we included age and gender as independent variables as well and thus, we 
TABLE 4: Various parameters associated with $\Delta$ urinary albumin excretion $(\triangle \mathrm{UAE})$ in univariate analyses.

\begin{tabular}{lcc}
\hline Comparison with baseline value & $\rho$ & $p$ \\
Age (years) & -0.2571 & n.s. \\
Gender & -0.1770 & n.s. \\
Baseline systolic blood pressure $(\mathrm{mmHg})$ & -0.3006 & 0.038 \\
Baseline urinary $\mathrm{Na}^{+}$excretion $(\mathrm{mEq} / \mathrm{L})$ & 0.1419 & n.s. \\
Baseline HbAlc $(\%)$ & -0.3073 & 0.027 \\
\hline Comparison with change of each value & $\rho$ & $p$ \\
Age (years) & -0.2571 & n.s. \\
Gender & -0.1770 & n.s. \\
$\Delta$ Systolic blood pressure $(\mathrm{mmHg})$ & 0.4660 & 0.0008 \\
$\Delta$ Urinary Na $^{+}$excretion $(\mathrm{mEq} / \mathrm{L})$ & -0.0428 & n.s. \\
$\Delta$ HbAlc $(\%)$ & 0.0659 & n.s. \\
\hline
\end{tabular}

TABLE 5: Determinant factors associated with $\Delta$ urinary albumin excretion $(\triangle \mathrm{UAE})$ in multivariate analyses.

\begin{tabular}{lcc}
\hline Explanatory variables & $\beta$ & $p$ \\
\hline Age (years) & 0.0726 & n.s. \\
Gender & 0.0502 & n.s. \\
Baseline systolic blood pressure $(\mathrm{mmHg})$ & -0.0222 & n.s. \\
Baseline HbA1c $(\%)$ & -0.166 & n.s. \\
$\Delta$ Systolic blood pressure & 0.417 & 0.01 \\
\hline
\end{tabular}

performed multivariate analysis using age, gender, sBP, and $\mathrm{HbAlc}$ levels at baseline and $\triangle \mathrm{sBP}$ as explanatory variables and $\triangle \mathrm{UAE}$ as an objective variable. As shown in Table 5, even after adjustment with age and gender, $\Delta \mathrm{sBP}$ was an independent factor contributing to the reduction of UAE after combination therapy.

\section{Discussion}

In this study, we showed that canagliflozin as add-on therapy to teneligliptin exerted beneficial effects on metabolic parameters such as body weight, $\mathrm{HbAlc}$, and uric acid without any severe adverse events (Table 1), which were compatible with the previous reports about the effectiveness of SGLT2 inhibitors [10-12]. In addition, in the univariate analysis, HbAlc levels at baseline were strongly associated with $\triangle \mathrm{HbAlc}$ (data not shown). Unexpectedly, lipid profile showed no significant improvement in this study (Table 1). As the lipid profile at baseline was relatively good in this study, it might have been hard to observe further improvement. It is also possible that as this study was retrospective, we could not educate exercise and/or diet adequately.

In this study, it is hard to directly compare the teneligliptin continued group and canagliflozin add-on group as participants' baseline characteristics were totally different. As canagliflozin was added on by each doctor's judgement, teneligliptin might be continued for patients whose HbAlc was relatively good and body weight was relatively low. It is true that there are some data defects such as fat mass, skeletal muscle mass, IRI, glucagon, IRI/glucagon ratio, ketone body, and urinary albumin excretion as this is a retrospective study. However, there were no other parameter changes after 3 months; we assume that there would be no change in parameters which were not measured in this study. On the contrary, we also explored the changes of various parameters between baseline and 3 months later (Table 2). As expected, the difference of changes such as body weight, BMI, FPG, and HbAlc was significantly larger in the canagliflozin add-on group. In addition, the difference of changes of Cre and eGFR became significant, which was not significantly different in each group comparison (Table 1). It is known that an initial eGFR drop is observed when SGLT2 inhibitors are used. This might partially explain the significant changes of Cre and eGFR in our study. However, unexpectedly, no significant difference in the changes of systolic and diastolic blood pressure was observed in our study. This might derive from some difference of the patients' background of each group or limited small sample size.

In general, it is said that SGLT2 inhibitors decrease serum insulin levels and increase serum glucagon levels, which lead to decrease of the insulin/glucagon ratio. However, in this study, IRI was not reduced after 3 months of combination therapy and there was no change in the IRI/glucagon ratio. There are some possible hypotheses that IRI was not decreased in this study. First, there were some patients who were recovering from glucose toxicity after adding canagliflozin. In these subjects, IRI levels were increased drastically. As sample size was rather small in this study, these subjects might have influenced the total IRI average. Second, as this study is retrospective, the difference of fasting time of each patient might have led to this result.

On the contrary, the serum glucagon level was significantly elevated after starting canagliflozin as add-on therapy to teneligliptin. Furthermore, the univariate analysis revealed $\Delta$ glucagon was not significantly associated with $\mathrm{HbA1c}$, IRI, and FPG at baseline and $\triangle \mathrm{HbAlc}, \triangle \mathrm{IRI}$, and $\triangle \mathrm{FPG}$. These results suggested that the increase of glucagon had no or little impact on glycemic control. Several previous studies reported that treatment with an SGLT2 inhibitor for 1-12 weeks significantly increased fasting or postprandial plasma glucagon levels in type 2 diabetes patients [13-15]. On the contrary, there are some reports which suggest that canagliflozin does not increase the serum glucagon level during meal tolerance test $[8,16]$. In addition, it is also presented that SGLT1 but not SGLT2 is expressed in the murine $\alpha \mathrm{TC} 1$ cell line and human and murine pancreatic islets and that canagliflozin reduced glucagon secretion from $\alpha \mathrm{TC1}$ cells in an SGLT1-dependent manner [17]. On the other hand, it is also reported that SGLT2 but not SGLT1 is expressed in glucagon-secreting alpha cells of human and murine pancreatic islets and dapagliflozin triggers glucagon secretion [18]. From the results of these reports, it is possible that some drug effects exist due to the difference of each drug affinity to SGLT1 and SGLT2. It will need further investigation to elucidate whether there are some drug effects on the changes of glucose metabolism and glucagon secretion. Although it is still controversial, we think it is important that our study 
showed that serum glucagon change did not affect overall metabolic changes in an adverse manner.

Noda et al. reported that teneligliptin and teneligliptin/canagliflozin combination therapy improved blood glucose levels after meal tolerance test (MTT) in 12 participants in a hospitalized setting for 14 days [16]. Interestingly, absolute values of glucagon and active GLP-1 were elevated after starting canagliflozin combination therapy, while $\Delta$ glucagon was significantly reduced and $\Delta$ active GLP-1 was increased during MTT compared to teneligliptin monotherapy. This study presented that canagliflozin actually increased glucagon levels, which was similar to our study. However, $\Delta$ glucagon during MTT was significantly reduced, which could not be confirmed in our study as we did not conduct MTT.

Okahata et al. reported that canagliflozin and canagliflozin/teneligliptin combination therapy improved blood glucose levels after MTT in 26 participants in a hospitalized setting for 6 days [19]. This study presented that canagliflozin increased that serum glucagon level during MTT and addition of teneligliptin reversed this increase. They also evaluated the change of the IRI/glucagon ratio during MTT, which resulted in no difference between canagliflozin treatment and canagliflozin/teneligliptin treatment.

These two studies suggest that addition of canagliflozin actually increases the serum glucagon level. Furthermore, the latter study presented no IRI/glucagon ratio difference during MTT between canagliflozin therapy and canagliflozin/teneligliptin combination therapy. On the contrary, these studies are in an inpatient setting, and the intervention term was quite short, and patients' backgrounds such as age and disease duration are totally different from our study.

Kadowaki et al. reported two large randomized control studies regarding the canagliflozin/teneligliptin combination vs. the monotherapy $[8,9]$. One was canagliflozin added on to teneligliptin. The other was vice versa. Canagliflozin added on to teneligliptin improved glucose levels during MTT. However, the serum glucagon level was not increased in this study. This result might have derived from the method of glucagon measurements. In their study, glucagon was measured by radioimmunoassay (RIA). It is reported that there is crossreactivity against several proglucagon-derived fragments in RIA for glucagon determination. Some reports have elucidated a large difference in glucagon results in the same sample between RIA and sandwich ELISA [20, 21]. This might partially explain the difference of the glucagon result from our study. Anyway, all studies including our study presented that canagliflozin add-on therapy to teneligliptin improved various metabolic parameters.

Next, in this study, we presented that $\triangle \mathrm{UAE}$ was significantly related to $\mathrm{sBP}$ and $\mathrm{HbA1c}$ levels at baseline and $\triangle \mathrm{sBP}$. Furthermore, multivariate analysis showed that $\triangle \mathrm{sBP}$ was an independent factor contributing to the reduction of UAE after combination therapy. Several large clinical trials reported that SGLT2 inhibitors such as empagliflozin, canagliflozin, and dapagliflozin reduced the incidence of end-stage kidney disease, doubling of the serum creatinine levels from baseline, or death from renal or cardiovascular disease [5-7]. Although there was no change in eGFR after starting combination therapy in this study, such $\triangle \mathrm{sBP}$ - dependent reduction of UAE might lead to a renal protective effect of canagliflozin add-on therapy in the long term.

Needless to say, there are limitations in this study. First, since this study was retrospective and our sample size was small, a large-scale and randomized prospective study would be necessary to achieve higher levels of evidence. Second, since most of the subjects in this study had mild to moderate renal dysfunction, the data obtained in this study is not necessarily true for subjects with severe renal dysfunction. Third, since these study subjects were middle aged and relatively obese, the data obtained in this study is not necessarily true for relatively aged and lean subjects. In addition, it is known that dual-energy X-ray absorptiometry is a gold standard method for the evaluation of body composition, but in this study, we used bioelectric impedance assessments to evaluate body composition because of their simplicity and portability.

Taken together, treatment with canagliflozin in addition to teneligliptin showed beneficial effects on various metabolic parameters and significantly reduced urinary albumin excretion. Furthermore, multivariate analyses showed that the reduction of urinary albumin excretion was mainly determined by the decrease of blood pressure. In addition, the change of serum glucagon levels was not related to any metabolic parameters, suggesting that the increase of glucagon levels after adding canagliflozin did not mainly, if any, contribute to the aggravation of overall metabolism. In conclusion, treatment with canagliflozin in addition to teneligliptin decreases urinary albumin excretion, at least in part, through the reduction of blood pressure and improves a variety of metabolic parameters in a glucagon-independent manner in Japanese subjects with type 2 diabetes mellitus.

\section{Data Availability}

The datasets used and/or analyzed during the current study are available from the corresponding author on reasonable request.

\section{Conflicts of Interest}

Hideaki Kaneto has received honoraria for lectures and received scholarship grants from Sanofi, Novo Nordisk, Lilly, Boehringer Ingelheim, MSD, Takeda, Ono Pharma, Daiichi Sankyo, Sumitomo Dainippon Pharma, Mitsubishi Tanabe Pharma, Pfizer, Kissei Pharma, AstraZeneca, Astellas, Novartis, Kowa, Chugai Pharmaceutical, and Taisho Pharmaceutical. Kohei Kaku has been an advisor to, received honoraria for lectures from, and received scholarship grants from Novo Nordisk, Sanwa Kagaku Kenkyusho, Takeda, Taisho Pharmaceutical, MSD, Kowa, Sumitomo Dainippon Pharma, Novartis, Mitsubishi Tanabe Pharma, AstraZeneca, Nippon Boehringer Ingelheim, Chugai Pharmaceutical, Daiichi Sankyo, and Sanofi. All other authors declare that they have no conflicts of interest.

\section{References}

[1] D. J. Drucker and M. A. Nauck, "The incretin system: glucagon-like peptide-1 receptor agonists and dipeptidyl 
peptidase-4 inhibitors in type 2 diabetes," The Lancet, vol. 368, no. 9548, pp. 1696-1705, 2006.

[2] A. A. Tahrani, A. H. Barnett, and C. J. Bailey, "SGLT inhibitors in management of diabetes," The Lancet Diabetes and Endocrinology, vol. 1, no. 2, pp. 140-151, 2013.

[3] H. Kaneto, A. Obata, M. Shimoda et al., "Promising diabetes therapy based on the molecular mechanism for glucose toxicity: usefulness of SGLT2 inhibitors as well as incretin-related drugs," Current Medicinal Chemistry, vol. 23, no. 27, pp. 3044-3051, 2016.

[4] H. Kaneto, A. Obata, T. Kimura et al., "Beneficial effects of sodium-glucose cotransporter 2 inhibitors for preservation of pancreatic $\beta$-cell function and reduction of insulin resistance," Journal of Diabetes, vol. 9, no. 3, pp. 219-225, 2017.

[5] B. Zinman, C. Wanner, J. M. Lachin et al., "Empagliflozin, cardiovascular outcomes, and mortality in type 2 diabetes," The New England Journal of Medicine, vol. 373, no. 22, pp. 21172128, 2015.

[6] B. Neal, V. Perkovic, K. W. Mahaffey et al., "Canagliflozin and cardiovascular and renal events in type 2 diabetes," The New England Journal of Medicine, vol. 377, no. 7, pp. 644-657, 2017.

[7] S. D. Wiviott, I. Raz, M. P. Bonaca et al., "Dapagliflozin and cardiovascular outcomes in type 2 diabetes," The New England Journal of Medicine, vol. 380, no. 4, pp. 347-357, 2019.

[8] T. Kadowaki, N. Inagaki, K. Kondo et al., "Long-term safety and efficacy of Canagliflozin as add-on therapy to teneligliptin in Japanese patients with type 2 diabetes," Diabetes, Obesity \& Metabolism, vol. 20, no. 1, pp. 77-84, 2018.

[9] T. Kadowaki, N. Inagaki, K. Kondo et al., "Efficacy and safety of teneligliptin added to canagliflozin monotherapy in Japanese patients with type 2 diabetes mellitus: a multicentre, randomized, double-blind, placebo-controlled, parallelgroup comparative study," Diabetes, Obesity \& Metabolism, vol. 20, no. 2, pp. 453-457, 2018.

[10] T. Kinoshita, M. Shimoda, J. Sanada et al., "There is a close association between the recovery of liver injury and glycemic control after SGLT2 inhibitor treatment in Japanese subjects with type 2 diabetes: a retrospective clinical study," Diabetes Therapy, vol. 9, no. 4, pp. 1569-1580, 2018.

[11] S. Kamei, M. Iwamoto, M. Kameyama et al., "Effect of tofogliflozin on body composition and glycemic control in Japanese subjects with type 2 diabetes mellitus," Journal Diabetes Research, vol. 2018, article 6470137, pp. 1-6, 2018.

[12] T. Kimura, J. Sanada, M. Shimoda et al., "Switching from lowdose thiazide diuretics to sodium-glucose cotransporter 2 inhibitor improves various metabolic parameters without affecting blood pressure in patients with type 2 diabetes and hypertension," Journal of Diabetes Investigation, vol. 9, no. 4, pp. 875-881, 2018.

[13] E. Ferrannini, E. Muscelli, S. Frascerra et al., "Metabolic response to sodium-glucose cotransporter 2 inhibition in type 2 diabetic patients," The Journal of Clinical Investigation, vol. 124, no. 2, pp. 499-508, 2014.

[14] W. Alsalim, M. Persson, and B. Ahrén, "Different glucagon effects during DPP-4 inhibition versus SGLT-2 inhibition in metformin-treated type 2 diabetes patients," Diabetes, Obesity \& Metabolism, vol. 20, no. 7, pp. 1652-1658, 2018.

[15] R. Martinez, H. al-Jobori, A. M. Ali et al., "Endogenous glucose production and hormonal changes in response to canagliflozin and liraglutide combination therapy," Diabetes, vol. 67, no. 6, pp. 1182-1189, 2018.

[16] T. Noda, E. Ebihara, H. Ueno et al., "Concurrent use of teneligliptin and canagliflozin improves glycemic control with beneficial effects on plasma glucagon and glucagon-like peptide-1: a single-arm study," Diabetes Therapy, vol. 10, no. 5, pp. 18351846, 2019.

[17] T. Suga, O. Kikuchi, M. Kobayashi et al., "SGLT1 in pancreatic $\alpha$ cells regulates glucagon secretion in mice, possibly explaining the distinct effects of SGLT2 inhibitors on plasma glucagon levels," Molecular Metabolism, vol. 19, pp. 1-12, 2019.

[18] C. Bonner, J. Kerr-Conte, V. Gmyr et al., "Inhibition of the glucose transporter SGLT2 with dapagliflozin in pancreatic alpha cells triggers glucagon secretion," Nature Medicine, vol. 21, no. 5, pp. 512-517, 2015.

[19] S. Okahata, K. Sakamoto, T. Mitsumatsu, Y. Kondo, S. Tanaka, and T. Shiba, "Mechanistic insights from sequential combination therapy with a sodium glucose co-transporter-2 inhibitor and a dipeptidyl peptidase-4 inhibitor: results from the CANARIS trial using canagliflozin and teneligliptin," Diabetes, Obesity \& Metabolism, vol. 21, no. 2, pp. 388-392, 2019.

[20] T. Matsuo, J. Miyagawa, Y. Kusunoki et al., "Postabsorptive hyperglucagonemia in patients with type 2 diabetes mellitus analyzed with a novel enzyme-linked immunosorbent assay," Journal of Diabetes Investigation, vol. 7, no. 3, pp. 324-331, 2016.

[21] A. Miyachi, M. Kobayashi, E. Mieno et al., "Accurate analytical method for human plasma glucagon levels using liquid chromatography-high resolution mass spectrometry: comparison with commercially available immunoassays," Analytical and Bioanalytical Chemistry, vol. 409, no. 25, pp. 5911-5918, 2017. 